\title{
A Sufficient Condition for Private Information Hiding of Two Correlated Sources Under Multiple Access Attacks*
}

\author{
Yadong Wang, Yangfan Zhong, Fady Alajaji and Tamás Linder \\ Department of Mathematics and Statistics \\ Queen's University, Kingston, ON, K7L 3N6, Canada \\ Email: \{yadong, yangfan, fady, linder\}@ mast.queensu.ca
}

\begin{abstract}
Consider a multi-user private information-hiding scenario in which two information hiders separately embed correlated sources $\left(S_{1}, S_{2}\right)$ into a common host source $U$ (covertext). The $i$-th information hider embeds the secret source $S_{i}$ into the covertext $U$ subject to a distortion constraint $D_{i}$ $(i=1,2)$. The outputs (stegotexts) are corrupted by a multiple access channel attack $W_{Y \mid X_{1} X_{2}}$. A sufficient condition (in singleletter form) under which $\left(S_{1}, S_{2}\right)$ can be successfully embedded into $U$ under $W_{Y \mid X_{1} X_{2}}$ is established.
\end{abstract}

\section{INTRODUCTION}

Information hiding is the means to embed a secret message into a host message (covertext) so that the information hider is able to transmit the information even though the transmission is subject to manipulation by an attacker attempting to render the hidden information undetectable. A large body of literature including theoretical studies as well as various practical applications have recently been devoted to this new area (see, e.g., [1]-[8] and the references therein).

In the literature, the information-hiding scenario is usually modeled as a constrained channel coding problem. The secret messages, assumed to be uniformly distributed over a given message set, are one-by-one embedded into the host messages. Since the secret messages should not interfere perceptually with the host messages, a distortion constraint is placed between the encoder output and the original host messages. From an information-theoretic point of view, the problem is to find the largest embedding rate (known as embedding capacity) for which, at the encoder, the distortion between the host messages and the output (stegotexts) does not exceed a preset threshold, and at the decoder, the secret messages can be reproduced with an arbitrarily small probability of error.

In practical situations (e.g., instant (online) data-hiding), in order to reduce the complexity of coding, we may need to directly hide an information source (or correlated sources) with a nonuniform distribution. In this work we extend the pointto-point information-hiding model to a multi-user setting. Our model is depicted in Fig. 1. Instead of embedding uniformly distributed indices, two encoders independently embed two (arbitrarily distributed) discrete memoryless correlated sources $\left(S_{1}, S_{2}\right)$ into a common memoryless host source $U$, and transmit the resulting sequences to a common destination in

\footnotetext{
*This work was supported in part by NSERC of Canada
}

the presence of discrete memoryless multiple access channel (MAC) attacks. One possible application of this scenario is that two agents separately embed noisy observations of the same source, and transmit the hidden information over a MAC attack channel. Throughout the paper, we deal with private information hiding; i.e., we assume that the decoder has perfect knowledge of $U$.

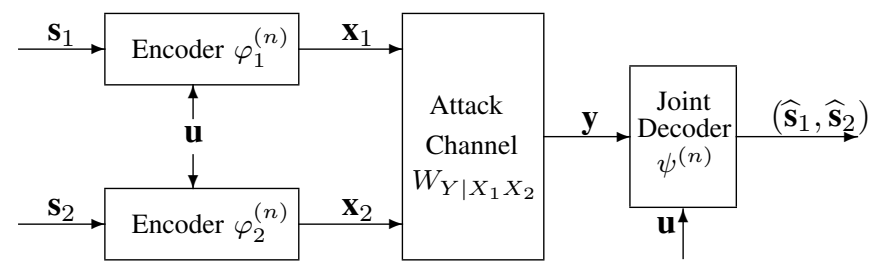

Fig. 1. A joint source coding and embedding model for multi-user information hiding.

Given the secret sources $\left(S_{1}, S_{2}\right)$, a MAC $W_{Y \mid X_{1} X_{2}}$, the host source $U$, and a distortion level pair $\left(D_{1}, D_{2}\right)$, one may ask whether there exists a coding scheme, such that $\left(S_{1}, S_{2}\right)$ can be embedded in $U$ within distortion levels $\left(D_{1}, D_{2}\right)$, and transmitted over $W_{Y \mid X_{1} X_{2}}$ with an arbitrarily small probability of error. To begin, we note that, especially in a multi-user system, jointly source coding and embedding the sources $\left(S_{1}, S_{2}\right)$ into $U$ might perform better than the traditional separate coding (i.e., concatenating lossless data compression and embedding). In this paper we investigate whether $\left(S_{1}, S_{2}\right)$ can be successfully transmitted under the MAC attacks by joint source coding and embedding codes. In particular, we establish a sufficient condition for successfully embedding $\left(S_{1}, S_{2}\right)$ into $U$ under the MAC $W_{Y \mid X_{1} X_{2}}$; see Theorem 1. Since our model jointly deals with information embedding (privacy protection) and compression of (nonuniform) correlated secret sources in a multiuser setting, it adds a new dimension to the traditional point-to-point information hiding problem. Note also that our problem can be viewed as a generalization of the problem of transmitting correlated sources over ordinary MAC channels [9]-[12].

\section{PRELIMINARIES}

Throughout, random variables (RV's) are denoted by capital letters, e.g., $X$, specific values are denoted by lower case letters, e.g., $x$, and their alphabets are denoted by calligraphic 
letters, e.g., $\mathcal{X}$. Similarly, random vectors are denoted by capital letters superscripted by their lengths, e.g., $X^{n}$, their alphabets are denoted by calligraphic letters superscripted by their lengths, e.g., $\mathcal{X}^{n}$, and their realizations are denoted by boldface lower case letters, e.g., $\mathbf{x} \triangleq\left(x_{1}, x_{2}, \ldots, x_{n}\right) \in \mathcal{X}^{n}$. The cardinality of a finite set $\mathcal{X}$ is denoted by $|\mathcal{X}| . \mathbb{E}(X)$ denotes the expectation of $X$. For any $\operatorname{RV} X, P_{X}(x)$ denotes the probability that $X=x$. For jointly distributed RV's $X$ and $U, P_{X \mid U}(x \mid u)$ denotes the conditional probability of $X=x$ given that $U=u$. The probability of an independent and identically distributed (i.i.d.) sequence $\mathbf{x} \in \mathcal{X}^{n}$ is given by $P_{X^{n}}(\mathbf{x}) \triangleq \prod_{j=1}^{n} P_{X}\left(x_{j}\right)$. All alphabets are finite, and all logarithms are in natural base.

Let $V \triangleq\left(X_{1}, X_{2}, \ldots, X_{m}\right)$ be a superletter (an ordered collection of RV's) taking values in a finite set $\mathcal{V} \triangleq$ $\mathcal{X}_{1} \times \mathcal{X}_{2} \times \cdots \times \mathcal{X}_{m}$ with joint distribution $P_{V}\left(x_{1}, \ldots, x_{m}\right)$, which for simplicity we also denote by $P_{V}(v)$. Denote by $T_{\epsilon}^{(n)}(V)$ or $T_{\epsilon}^{(n)}$ the set of all strongly $\epsilon$-typical sequences $\left(\mathbf{x}_{1}, \ldots, \mathbf{x}_{m}\right)$ [13] with respect to the joint distribution $P_{V}(v)$. Let $I_{V} \triangleq\{1,2, \ldots, m\}$, and $I_{G} \subseteq I_{V}$. We then let $G=\left(X_{g_{1}}, X_{g_{2}}, \ldots, X_{g_{\left|I_{G}\right|}}\right) \in \mathcal{G}$ be a "subsuperletter" corresponding to $I_{G}$ such that $g_{i} \in I_{G}$. Let $G$, $K$, and $L$ be sub-superletters of $V$ such that $I_{G}, I_{K}, I_{L}$ are disjoint, and let $P_{G}, P_{K}$ and $P_{G \mid K}$ be the marginal and conditional distributions induced by $P_{V}$, respectively. Denote by $T_{\epsilon}^{(n)}(G)$ the restriction of $T_{\epsilon}^{(n)}(V)$ to the coordinates of $G$ [13]. Given $\mathbf{k} \in T_{\epsilon}^{(n)}(K)$, denote $T_{\epsilon}^{(n)}(G \mid \mathbf{k}) \triangleq$ $\left\{\mathbf{g} \in \mathcal{G}^{n}:(\mathbf{g}, \mathbf{k}) \in T_{\epsilon}^{(n)}(G, K)\right\} \cdot T_{\epsilon}^{(n)}(G \mid \mathbf{k})$ is sometimes referred to as $T_{\epsilon}^{(n)}(\cdot \mid \mathbf{k})$ if $G$ is clear from the context.

Lemma 1: [13, pp. 342-343] Let $G^{n}, K^{n}$ and $V^{n}$ be i.i.d. drawn according to $P_{G^{n}}, P_{K^{n}}$ and $P_{V^{n}}$. The following properties hold for sufficiently large $n$.

1) $P_{V^{n}}\left(\left\{V^{n} \in T_{\epsilon}^{(n)}\right\}\right) \geq 1-\epsilon$, and

$$
P_{K^{n}}\left(\left\{K^{n} \in T_{\epsilon}^{(n)}(K)\right\}\right) \geq 1-\epsilon .
$$

2) For any $\mathbf{k} \in T_{\epsilon}^{(n)}(K)$,

$$
\left|(1 / n) \log P_{K^{n}}(\mathbf{k})+H(K)\right| \leq \epsilon .
$$

3) $(1-\epsilon) e^{n(H(K)-\epsilon)} \leq\left|T_{\epsilon}^{(n)}(K)\right| \leq e^{n(H(K)+\epsilon)}$.

4) For any $\mathbf{k} \in T_{\epsilon}^{(n)}(K)$,

$$
P_{G^{n}}\left(\left\{G^{n} \in T_{\epsilon}^{(n)}(G \mid \mathbf{k})\right\}\right) \geq 1-\epsilon .
$$

5) For any $\mathbf{k} \in T_{\epsilon}^{(n)}(K)$,

$$
(1-\epsilon) e^{n(H(G \mid K)-2 \epsilon)} \leq\left|T_{\epsilon}^{(n)}(G \mid \mathbf{k})\right| \leq e^{n(H(G \mid K)+2 \epsilon)} .
$$

6) For $(\mathbf{g}, \mathbf{k}) \in T_{\epsilon}^{(n)}(G, K)$,

$$
\left|(1 / n) \log P_{G^{n} \mid K^{n}}(\mathbf{g} \mid \mathbf{k})+H(G \mid K)\right| \leq 2 \epsilon .
$$

Lemma 2: (Markov Lemma [13, p. 579]) Let $G \rightarrow K \rightarrow$ $L$ form a Markov chain in this order. For $0<\epsilon<1$ and $(\mathbf{g}, \mathbf{k}) \in T_{\epsilon}^{(n)}(G, K)$, if $L^{n}$ is i.i.d. drawn given $k$ according to $\prod_{j=1}^{n} P_{L \mid K}\left(l_{j} \mid k_{j}\right)$, then $\operatorname{Pr}\left\{\left(\mathbf{g}, \mathbf{k}, L^{n}\right) \in T_{\epsilon}^{(n)}(G, K, L)\right\}>$ $1-\epsilon$ for $n$ sufficiently large.

\section{Problem Formulation and Main Results}

Let the pair of memoryless correlated secret sources $\left\{\left(S_{1 j}, S_{2 j}\right)\right\}_{j=1}^{\infty}$ have marginal distribution $P_{S_{1} S_{2}}$ and denote the marginal distribution of the host source $\left\{U_{j}\right\}_{j=1}^{\infty}$ by $P_{U}$. Assume $\left(S_{1}, S_{2}\right)$ and $U$ are independent. The attack channel is modeled as a two-sender one-receiver discrete memoryless MAC $W_{Y \mid X_{1} X_{2}}$ having input alphabets $\mathcal{X}_{1}$ and $\mathcal{X}_{2}$, output alphabet $\mathcal{Y}$, and a transition probability distribution $W_{Y \mid X_{1} X_{2}}\left(y \mid x_{1}, x_{2}\right)$. The probability of receiving $\mathbf{y} \in \mathcal{Y}^{n}$ conditioned on sending $\mathbf{x}_{1} \in \mathcal{X}_{1}^{n}$ and $\mathbf{x}_{2} \in \mathcal{X}_{2}^{n}$ is hence given by $W_{Y^{n} \mid X_{1}^{n} X_{2}^{n}}\left(\mathbf{y} \mid \mathbf{x}_{1}, \mathbf{x}_{2}\right)=\prod_{j=1}^{n} W_{Y \mid X_{1} X_{2}}\left(y_{j} \mid x_{1 j}, x_{2 j}\right)$. Let $d_{i}: \mathcal{U} \times \mathcal{X}_{i} \rightarrow[0, \infty)$ be single-letter distortion measures and define $d_{i}^{\max } \triangleq \max _{u, x_{i}} d_{i}\left(u, x_{i}\right)$ for $i=1,2$. For $\mathbf{u} \in \mathcal{U}^{n}$ and $\mathbf{x}_{i} \in \mathcal{X}_{i}^{n}$, let $d_{i}\left(\mathbf{u}, \mathbf{x}_{i}\right)=\sum_{j=1}^{n} d_{i}\left(u_{j}, x_{i j}\right)$.

A joint source coding and embedding (JSCE) code $\left(\varphi_{1}^{(n)}, \varphi_{2}^{(n)}, \psi^{(n)}\right)$ with block length $n$ consists of two encoders $\varphi_{1}^{(n)}: \mathcal{S}_{1}^{n} \times \mathcal{U}^{n} \rightarrow \mathcal{X}_{1}^{n}$ and $\varphi_{2}^{(n)}: \mathcal{S}_{2}^{n} \times \mathcal{U}^{n} \rightarrow \mathcal{X}_{2}^{n}$ and a decoder $\psi^{(n)}: \mathcal{Y}^{n} \times \mathcal{U}^{n} \rightarrow \mathcal{S}_{1}^{n} \times \mathcal{S}_{2}^{n}$; see Fig. 1. The probability of error in reproducing the secret sources is given by

$$
\begin{aligned}
& P_{e}^{(n)}= \sum_{\mathcal{S}_{1}^{n} \times \mathcal{S}_{2}^{n} \times \mathcal{U}^{n}} P_{S_{1}^{n} S_{2}^{n}}\left(\mathbf{s}_{1}, \mathbf{s}_{2}\right) P_{U^{n}}(\mathbf{u}) \\
& \sum_{\mathbf{y}: \psi^{(n)}(\mathbf{y}, \mathbf{u}) \neq\left(\mathbf{s}_{1}, \mathbf{s}_{2}\right)} W_{Y^{n} \mid X_{1}^{n} X_{2}^{n}}\left(\mathbf{y} \mid \mathbf{x}_{1}, \mathbf{x}_{2}\right)
\end{aligned}
$$

where $\mathbf{x}_{i} \triangleq \varphi_{i}^{(n)}\left(\mathbf{s}_{i}, \mathbf{u}\right)(i=1,2)$.

Definition 1: Given $P_{U}$ and distortion levels $D_{1}>0$ and $D_{2}>0$, we say that the secret sources $\left\{\left(S_{1 j}, S_{2 j}\right)\right\}$ are $\left(D_{1}, D_{2}\right)$-admissible with respect to the MAC $W_{Y \mid X_{1} X_{2}}$, if there exists a sequence of codes $\left(\varphi_{1}^{(n)}, \varphi_{2}^{(n)}, \psi^{(n)}\right)$ such that $P_{e}^{(n)} \rightarrow 0$ as $n \rightarrow \infty$ and for any $\delta>0, \frac{1}{n} \mathbb{E}\left[d_{i}\left(U^{n}, X_{i}^{n}\right)\right] \leq$ $D_{i}+\delta, i=1,2$, for $n$ sufficiently large.

Theorem 1: $\left\{\left(S_{1 j}, S_{2 j}\right)\right\}$ are $\left(D_{1}, D_{2}\right)$-admissible with respect to the MAC $W_{Y \mid X_{1} X_{2}}$ if there exist some RV $Q$ and a pair of conditional distributions $\left(P_{X_{1} \mid S_{1} U Q}, P_{X_{2} \mid S_{2} U Q}\right)$ such that

$$
\begin{aligned}
& H\left(S_{1} \mid S_{2}\right)<I\left(X_{1} ; Y \mid X_{2}, S_{2}, U, Q\right), \\
& H\left(S_{2} \mid S_{1}\right)<I\left(X_{2} ; Y \mid X_{1}, S_{1}, U, Q\right), \\
& H\left(S_{1}, S_{2}\right)<I\left(X_{1}, X_{2} ; Y \mid U, Q\right), \\
& \mathbb{E}\left[d_{i}\left(U, X_{i}\right)\right] \leq D_{i}, i=1,2,
\end{aligned}
$$

where the above entropies, mutual informations, and expectations are taken with respect to the joint distribution

$$
P_{Q} P_{S_{1} S_{2}} P_{U} P_{X_{1} \mid S_{1} U Q} P_{X_{2} \mid S_{2} U Q} W_{Y \mid X_{1} X_{2}} .
$$

We remark that the RV $Q$ serves as a time-sharing $\mathrm{RV}$ and the cardinality of its alphabet can be bounded by $|\mathcal{Q}| \leq 6$.

The proof of the theorem, which employs a joint strong typicality coding argument [9] under additional distortion constraints, is deferred to Section V. Note that if $U$ is removed in (1)-(3), then the inequalities reduce to the sufficient condition under which the sources $\left\{\left(S_{1 j}, S_{2 j}\right)\right\}$ can be reliably transmitted over the MAC $W_{Y \mid X_{1} X_{2}}$ obtained in [9], [10].

\section{Special Cases}

1) Uniform and Independent Sources: Suppose that the sources are independent and uniform, i.e., $P_{S_{1}}\left(s_{1}\right)=1 /\left|\mathcal{S}_{1}\right|$, 
$P_{S_{2}}\left(s_{2}\right)=1 /\left|\mathcal{S}_{2}\right|$ and $P_{S_{1} S_{2}}\left(s_{1}, s_{2}\right)=P_{S_{1}}\left(s_{1}\right) P_{S_{2}}\left(s_{2}\right)$ for any $\left(s_{1}, s_{2}\right) \in \mathcal{S}_{1} \times \mathcal{S}_{2}$. Define $\widetilde{R}_{1}=H\left(S_{1}\right)=\log \left|\mathcal{S}_{1}\right|$ and $\widetilde{R}_{2}=H\left(S_{2}\right)=\log \left|\mathcal{S}_{2}\right|$ to be the rates of the sources. By Theorem 1, $\left\{\left(S_{1 j}, S_{2 j}\right)\right\}$ are $\left(D_{1}, D_{2}\right)$-admissible with respect to the MAC $W_{Y \mid X_{1} X_{2}}$ if there exists some $\operatorname{RV} Q$ with $|\mathcal{Q}| \leq 6$, and a pair of distributions $\left(P_{X_{1} \mid U Q}, P_{X_{2} \mid U Q}\right)$ such that

$$
\begin{aligned}
& \widetilde{R}_{1}<I\left(X_{1} ; Y \mid X_{2}, U, Q\right), \\
& \widetilde{R}_{2}<I\left(X_{2} ; Y \mid X_{1}, U, Q\right), \\
& \widetilde{R}_{1}+\widetilde{R}_{2}<I\left(X_{1}, X_{2} ; Y \mid U, Q\right), \\
& \mathbb{E}\left[d_{i}\left(U, X_{i}\right)\right] \leq D_{i}, i=1,2,
\end{aligned}
$$

where the above mutual informations and expectations are taken with respect to the joint distribution $P_{Q} P_{U} P_{X_{1} \mid U Q} P_{X_{2} \mid U Q} W_{Y \mid X_{1} X_{2}}$. If we further set $D_{1} \geq d_{1}^{\max }$ and $D_{2} \geq d_{2}^{\max }$ and let $U$ be deterministic, inequalities (6)-(9) give the capacity region of the MAC [13].

2) Parallel Attack Channels: Assume that the attack MAC is composed of two independent discrete memoryless channels $W_{Y \mid X_{1} X_{2}}\left(y \mid x_{1}, x_{2}\right)=W_{Y_{1} \mid X_{1}}\left(y_{1} \mid x_{1}\right) \times W_{Y_{2} \mid X_{2}}\left(y_{2} \mid x_{2}\right)$ where $W_{Y_{i} \mid X_{i}}$ has input alphabet $\mathcal{X}_{i}$ and output alphabet $\mathcal{Y}_{i}$ such that $\mathcal{Y}_{1} \times \mathcal{Y}_{2}=\mathcal{Y}, i=1,2$. This can be interpreted as two attackers separately attacking the stegotexts. In this case, the condition given by Theorem 1 for successful embedding is equivalent to the following (see the proof in the appendix): $\left\{\left(S_{1 j}, S_{2 j}\right)\right\}$ are $\left(D_{1}, D_{2}\right)$-admissible with respect to the MAC $W_{Y \mid X_{1} X_{2}}$ if

$$
\begin{aligned}
& H\left(S_{1} \mid S_{2}\right)<C\left(W^{(1)}, D_{1}\right), \\
& H\left(S_{2} \mid S_{1}\right)<C\left(W^{(2)}, D_{2}\right), \\
& H\left(S_{1}, S_{2}\right)<C\left(W^{(1)}, D_{1}\right)+C\left(W^{(2)}, D_{2}\right),
\end{aligned}
$$

where $C\left(W^{(i)}, D_{i}\right)=\max _{\mathbb{E}}\left[d_{i}\left(U, X_{i}\right)\right] \leq D_{i} I\left(X_{i} ; Y_{i} \mid U\right), i=$ 1,2 is the information-hiding capacity of the attack channel $W_{Y_{i} \mid X_{i}}$ with distortion threshold $D_{i}$ [4].

3) Attack-Free Channel: Let $l: \mathcal{X}_{1} \times \mathcal{X}_{2} \rightarrow \mathcal{Y}$ be a bijection and let $Y=l\left(X_{1}, X_{2}\right)$. In this case, Theorem 1 implies that $\left\{\left(S_{1 j}, S_{2 j}\right)\right\}$ are $\left(D_{1}, D_{2}\right)$-admissible with respect to the MAC $W_{Y \mid X_{1} X_{2}}$ if

$$
\begin{aligned}
& H\left(S_{1} \mid S_{2}\right)<H\left(X_{1} \mid X_{2}, S_{2}, U\right), \\
& H\left(S_{2} \mid S_{1}\right)<H\left(X_{2} \mid X_{1}, S_{1}, U\right), \\
& H\left(S_{1}, S_{2}\right)<H\left(X_{1}, X_{2} \mid U\right), \\
& \mathbb{E}\left[d_{i}\left(U, X_{i}\right)\right] \leq D_{i}, i=1,2,
\end{aligned}
$$

where the entropies are taken under the joint distribution $P_{S_{1} S_{2}} P_{U} P_{X_{1} \mid S_{1} U} P_{X_{2} \mid S_{2} U}$. Note also that conditions (13)-(16) give the Slepian-Wolf lossless data compression region [13], [11] if we set $D_{1} \geq d_{1}^{\max }, D_{2} \geq d_{2}^{\max }$, and let $U$ be deterministic.

\section{Proof of Theorem 1}

We first give an outline of the proof. We need to show that for given $P_{S_{1} S_{2}}, P_{U}$, and $W_{Y \mid X_{1} X_{2}}$, there exists a sequence of JSCE codes $\left(\varphi_{1}^{(n)}, \varphi_{2}^{(n)}, \psi^{(n)}\right)$ such that $P_{e}^{(n)} \rightarrow 0$ as $n \rightarrow \infty$ and for any $\delta>0, \frac{1}{n} \mathbb{E}\left[d_{i}\left(U^{n}, X_{i}^{n}\right)\right] \leq D_{i}+\delta, i=1,2$, for $n$ sufficiently large. Fix $\left(P_{Q}, P_{X_{1} \mid S_{1} U Q}, P_{X_{2} \mid S_{2} U Q}\right)$ such that the following are satisfied for some $\epsilon>0$,

$$
\begin{aligned}
& H\left(S_{1} \mid S_{2}\right)<I\left(X_{1} ; Y \mid X_{2}, S_{2}, U, Q\right)-7 \epsilon, \\
& H\left(S_{2} \mid S_{1}\right)<I\left(X_{2} ; Y \mid X_{1}, S_{1}, U, Q\right)-7 \epsilon, \\
& H\left(S_{1}, S_{2}\right)<I\left(X_{1}, X_{2} ; Y \mid U, Q\right)-7 \epsilon, \\
& \mathbb{E}\left[d_{i}\left(U, X_{i}\right)\right] \leq D_{i}, i=1,2 .
\end{aligned}
$$

Define $P_{i}^{(n)} \triangleq \operatorname{Pr}\left\{d_{i}\left(U^{n}, X_{i}^{n}\right)>n\left(D_{i}+\epsilon\right)\right\}, i=1,2$. We will prove that for any $\epsilon_{1}>0$, the following probabilities, which are averaged over a family of random codes $\left(\mathcal{C}_{1}, \mathcal{C}_{2}\right)$, $i=1,2$, satisfy

$\mathbb{E}_{\mathcal{C}_{1}, \mathcal{C}_{2}}\left[P_{e}^{(n)}\right] \leq \epsilon_{1}, \quad \mathbb{E}_{\mathcal{C}_{1}, \mathcal{C}_{2}}\left[P_{i}^{(n)}\right] \leq \epsilon_{1}, \quad \mathbb{E}_{\mathcal{C}_{1}, \mathcal{C}_{2}}\left[P_{i}^{(n)}\right] \leq \epsilon_{1}$

for $n$ sufficiently large. Then $\mathbb{E}_{\mathcal{C}_{1}, \mathcal{C}_{2}}\left\{P_{e}^{(n)}+P_{1}^{(n)}+P_{2}^{(n)}\right\} \leq$ $3 \epsilon_{1}$, which guarantees that there exists at least one pair $\left(\mathcal{C}_{1}, \mathcal{C}_{2}\right)$ such that $P_{e}^{(n)}+P_{1}^{(n)}+P_{2}^{(n)} \leq 3 \epsilon_{1}$ and hence $P_{e}^{(n)} \leq 3 \epsilon_{1}$, $P_{1}^{(n)} \leq 3 \epsilon_{1}, P_{2}^{(n)} \leq 3 \epsilon_{1}$ are simultaneously satisfied for $n$ sufficiently large. Finally, it can be easily shown that $P_{i}^{(n)} \leq$ $3 \epsilon_{1}$ implies for $n$ sufficiently large that

$$
\frac{1}{n} \mathbb{E}\left[d_{i}\left(U^{n}, X_{i}^{n}\right)\right] \leq D_{i}+\epsilon+d_{i}^{\max } P_{i}^{(n)} \leq D_{i}+\delta .
$$

\section{A. Random Code Design}

Random Code Generation. Let $i \in\{1,2\}$. Choose a typical sequence $\mathbf{q}=\left(q_{1}, q_{2}, \ldots, q_{n}\right)$ arbitrarily in $T_{\epsilon}^{(n)}(Q)$. The sequence serves as a time sharing sequence and it is known at both the encoders and the decoder. For any sequences $\mathbf{s}_{i}, \mathbf{u}$ and the fixed $\mathbf{q}$, generate one $\mathbf{x}_{i}\left(\mathbf{s}_{i}, \mathbf{u}, \mathbf{q}\right)$ sequence according to $\prod_{j=1}^{n} P_{X_{i} \mid S_{i} U Q}\left(x_{i j} \mid s_{i j}, u_{j}, q_{j}\right)$. Define codebook $\mathcal{C}_{i}$ as $\mathcal{C}_{i} \triangleq$ $\left\{\mathbf{x}_{i}\left(\mathbf{s}_{i}, \mathbf{u}, \mathbf{q}\right):\left(\mathbf{s}_{i}, \mathbf{u}\right) \in \mathcal{S}_{i}^{n} \times \mathcal{U}^{n}\right\}$. Reveal the codebooks to both the encoders and the decoder.

Encoding. Given $\left(\mathbf{s}_{i}, \mathbf{u}\right) \in \mathcal{S}_{i}^{n} \times \mathcal{U}^{n}$, Encoder $i$ sends $\mathbf{x}_{i}\left(\mathbf{s}_{i}, \mathbf{u}, \mathbf{q}\right)$.

Decoding. The decoder has full knowledge of $\mathbf{u}$ (and also the time sharing sequence $\mathbf{q}$ ). Upon receiving sequence $\mathbf{y}$, the decoder finds the only pair $\left(\widehat{\mathbf{s}}_{1}, \widehat{\mathbf{s}}_{2}\right) \in T_{\epsilon}^{(n)}\left(S_{1}, S_{2}\right)$, such that $\mathbf{y} \in T_{\epsilon}^{(n)}\left(Y \mid \widehat{\mathbf{s}}_{1}, \widehat{\mathbf{s}}_{2}, \mathbf{u}, \mathbf{q}, \widehat{\mathbf{x}}_{1}, \widehat{\mathbf{x}}_{2}\right)$, where $\widehat{\mathbf{x}}_{1}=\mathbf{x}_{1}\left(\widehat{\mathbf{s}}_{1}, \mathbf{u}, \mathbf{q}\right)$ and $\widehat{\mathbf{x}}_{2}=\mathbf{x}_{2}\left(\widehat{\mathbf{s}}_{2}, \mathbf{u}, \mathbf{q}\right)$. If there is no or more than one such pair of sequences $\left(\widehat{\mathbf{s}}_{1}, \widehat{\mathbf{s}}_{2}\right)$, an error is declared.

For the sake of convenience, define the events

$$
\begin{array}{lll}
A_{0} \quad: \quad\left(\mathbf{s}_{1}, \mathbf{s}_{2}, \mathbf{u}\right) \in T_{\epsilon}^{(n)}\left(S_{1}, S_{2}, U \mid \mathbf{q}\right) \\
A_{1} \quad: \quad\left(\mathbf{s}_{1}, \mathbf{s}_{2}, \mathbf{u}, X_{1}^{n}\left(\mathbf{s}_{1}, \mathbf{u}, \mathbf{q}\right), X_{2}^{n}\left(\mathbf{s}_{2}, \mathbf{u}, \mathbf{q}\right)\right) \in T_{\epsilon}^{(n)}(\cdot \mid \mathbf{q}) .
\end{array}
$$

The following result is a consequence of the Markov lemma (Lemma 2).

Lemma 3: For any $\epsilon, \epsilon_{2} \in(0,1), \mathbb{E}_{\mathcal{C}_{1}, \mathcal{C}_{2}}\left[\operatorname{Pr}\left(A_{1}^{c} \mid A_{0}\right)\right] \leq \epsilon_{2}$ for $n$ sufficiently large, where the expectation is taken with respect to the random codes $\mathcal{C}_{1}$ and $\mathcal{C}_{2}$. 
B. Bounding $\mathbb{E}_{\mathcal{C}_{1}, \mathcal{C}_{2}}\left[P_{e}^{(n)}\right]$

$$
\begin{aligned}
\mathbb{E}_{\mathcal{C}_{1}, \mathcal{C}_{2}}\left[P_{e}^{(n)}\right] \leq & \sum_{\left(T_{\epsilon}^{(n)}\left(S_{1}, S_{2}, U \mid \mathbf{q}\right)\right)^{c}} P_{S_{1}^{n} S_{2}^{n}}\left(\mathbf{s}_{1}, \mathbf{s}_{2}\right) P_{U^{n}}(\mathbf{u}) \\
+ & \sum_{T_{\epsilon}^{(n)}\left(S_{1}, S_{2}, U \mid \mathbf{q}\right)} P_{S_{1}^{n} S_{2}^{n}}\left(\mathbf{s}_{1}, \mathbf{s}_{2}\right) P_{U^{n}}(\mathbf{u}) \\
& \times \mathbb{E}_{\mathcal{C}_{1}, \mathcal{C}_{2}}\left[\sum_{\mathbf{y}: \psi^{(n)}(\mathbf{y}, \mathbf{u}) \neq\left(\mathbf{s}_{1}, \mathbf{s}_{2}\right)} W_{\left.Y^{n} \mid X_{1}^{n} X_{2}^{n}\left(\mathbf{y} \mid \mathbf{x}_{1}, \mathbf{x}_{2}\right)\right] .}\right.
\end{aligned}
$$

The first term vanishes for $n$ sufficiently large by Lemma 1 . It suffices to bound the expectation in the second term. Given $\left(\mathbf{s}_{1}, \mathbf{s}_{2}, \mathbf{u}\right) \in T_{\epsilon}^{(n)}\left(S_{1}, S_{2}, U \mid \mathbf{q}\right)$, we have the following four error events:

$E_{0}:\left(\mathbf{s}_{1}, \mathbf{s}_{2}, \mathbf{u}, X_{1}^{n}\left(\mathbf{s}_{1}, \mathbf{u}, \mathbf{q}\right), X_{2}^{n}\left(\mathbf{s}_{2}, \mathbf{u}, \mathbf{q}\right), Y^{n}\right) \notin T_{\epsilon}^{(n)}(\cdot \mid \mathbf{q})$,

$E_{1}: \exists \quad \widehat{\mathbf{s}}_{1} \neq \mathbf{s}_{1}$ such that

$\left(\widehat{\mathbf{s}}_{1}, \mathbf{s}_{2}, \mathbf{u}, X_{1}^{n}\left(\widehat{\mathbf{s}}_{1}, \mathbf{u}, \mathbf{q}\right), X_{2}^{n}\left(\mathbf{s}_{2}, \mathbf{u}, \mathbf{q}\right), Y^{n}\right) \in T_{\epsilon}^{(n)}(\cdot \mid \mathbf{q})$, $E_{2}: \exists \widehat{\mathbf{s}}_{2} \neq \mathbf{s}_{2}$ such that

$\left(\mathbf{s}_{1}, \widehat{\mathbf{s}}_{2}, \mathbf{u}, X_{1}^{n}\left(\mathbf{s}_{1}, \mathbf{u}, \mathbf{q}\right), X_{2}^{n}\left(\widehat{\mathbf{s}}_{2}, \mathbf{u}, \mathbf{q}\right), Y^{n}\right) \in T_{\epsilon}^{(n)}(\cdot \mid \mathbf{q})$, $E_{3}: \exists \widetilde{\mathbf{s}}_{1} \neq \mathbf{s}_{1}, \widetilde{\mathbf{s}}_{2} \neq \mathbf{s}_{2}$ such that

$$
\left.\left(\widetilde{\mathbf{s}}_{1}, \widetilde{\mathbf{s}}_{2}, \mathbf{u}, X_{1}^{n}\left(\widetilde{\mathbf{s}}_{1}, \mathbf{u}, \mathbf{q}\right), \mathbf{u}\right), X_{2}^{n}\left(\widetilde{\mathbf{s}}_{2}, \mathbf{u}, \mathbf{q}\right), Y^{n}\right) \in T_{\epsilon}^{(n)}(\cdot \mid \mathbf{q}) .
$$

It then immediately follows from the union bound that

$$
\begin{gathered}
\mathbb{E}_{\mathcal{C}_{1}, \mathcal{C}_{2}}\left[\sum_{\mathbf{y}: \psi^{(n)}(\mathbf{y}, \mathbf{u}) \neq\left(\mathbf{s}_{1}, \mathbf{s}_{2}\right)} W_{Y^{n} \mid X_{1}^{n} X_{2}^{n}}\left(\mathbf{y} \mid \mathbf{x}_{1}, \mathbf{x}_{2}\right)\right] \\
\leq \sum_{j=0}^{3} \mathbb{E}_{\mathcal{C}_{1}, \mathcal{C}_{2}}\left[\operatorname{Pr}\left\{E_{j} \mid A_{0}\right\}\right] .
\end{gathered}
$$

To bound $\mathbb{E}_{\mathcal{C}_{1}, \mathcal{C}_{2}}\left[\operatorname{Pr}\left\{E_{0} \mid A_{0}\right\}\right]$, it follows from Lemmas 2 and 3 that

$$
\begin{aligned}
& \mathbb{E}_{\mathcal{C}_{1}, \mathcal{C}_{2}}\left[\operatorname{Pr}\left\{E_{0} \mid A_{0}\right\}\right] \\
& \leq \mathbb{E}_{\mathcal{C}_{1}, \mathcal{C}_{2}}\left[\operatorname{Pr}\left(A_{1}^{c} \mid A_{0}\right)\right]+\mathbb{E}_{\mathcal{C}_{1}, \mathcal{C}_{2}}\left[\operatorname{Pr}\left\{E_{0} \mid A_{0}, A_{1}\right\}\right] \\
& \leq \frac{\epsilon_{0}}{2}+\frac{\epsilon_{0}}{2}=\epsilon_{0}
\end{aligned}
$$

if $n$ sufficiently large, where $\epsilon_{0}$ will be specified later.

To bound $\mathbb{E}_{\mathcal{C}_{1}, \mathcal{C}_{2}}\left[\operatorname{Pr}\left\{E_{1} \mid A_{0}\right\}\right]$, write

$$
\begin{aligned}
& \mathbb{E}_{\mathcal{C}_{1}, \mathcal{C}_{2}}\left[\operatorname{Pr}\left\{E_{1} \mid A_{0}\right\}\right] \\
& \quad \leq \sum_{\widehat{\mathbf{s}}_{1} \neq \mathbf{s}_{1}: \widehat{\mathbf{s}}_{1} \in T_{\epsilon}^{(n)}\left(S_{1} \mid \mathbf{s}_{2}\right)} \mathbb{E}_{\mathcal{C}_{1}, \mathcal{C}_{2}}\left[\operatorname{Pr}\left\{\mathbf{v}_{1} \in T_{\epsilon}^{(n)} \mid A_{0}\right\}\right]
\end{aligned}
$$

where $\mathbf{v}_{1}=\left(\widehat{\mathbf{s}}_{1}, \mathbf{s}_{2}, \mathbf{u}, \mathbf{q}, X_{1}^{n}\left(\widehat{\mathbf{s}}_{1}, \mathbf{u}, \mathbf{q}\right), X_{2}^{n}\left(\mathbf{s}_{2}, \mathbf{u}, \mathbf{q}\right), Y^{n}\right)$ and the expectation can be upper bounded by

$$
\begin{aligned}
& \mathbb{E}_{\mathcal{C}_{1}, \mathcal{C}_{2}}\left[\operatorname{Pr}\left\{\mathbf{v}_{1} \in T_{\epsilon}^{(n)} \mid A_{0}\right\}\right] \\
& \leq \sum_{\mathcal{X}_{1}^{n} \times \mathcal{X}_{2}^{n}} P_{X_{1}^{n} \mid S_{1}^{n} U^{n} Q^{n}}\left(\widehat{\mathbf{x}}_{1} \mid \widehat{\mathbf{s}}_{1}, \mathbf{u}, \mathbf{q}\right) P_{X_{2}^{n} \mid S_{2}^{n} U^{n}}\left(\mathbf{x}_{2} \mid \mathbf{\mathbf { s } _ { 2 }}, \mathbf{u}, \mathbf{q}\right) \\
& \sum_{\mathbf{y} \in T_{\epsilon}^{(n)}\left(Y \mid \widehat{\mathbf{s}}_{1}, \mathbf{s}_{2}, \mathbf{u}, \mathbf{q}, \widehat{\mathbf{x}}_{1}, \mathbf{x}_{2}\right)} P_{Y^{n} \mid S_{2}^{n} U^{n} Q^{n} X_{2}^{n}\left(\mathbf{y} \mid \mathbf{s}_{2}, \mathbf{u}, \mathbf{q}, \mathbf{x}_{2}\right)}
\end{aligned}
$$

$$
\begin{aligned}
& \leq\left|T_{\epsilon}^{(n)}\left(Y \mid \widehat{\mathbf{s}}_{1}, \mathbf{s}_{2}, \mathbf{u}, \mathbf{q}, \widehat{\mathbf{x}}_{1}, \mathbf{x}_{2}\right)\right| e^{-n\left(H\left(Y \mid S_{2}, U, Q, X_{2}\right)-2 \epsilon\right.}(24) \\
& \leq e^{n\left(H\left(Y \mid X_{1}, X_{2}\right)+2 \epsilon\right)} e^{-n\left(H\left(Y \mid S_{2}, U, Q, X_{2}\right)-2 \epsilon\right)} \\
& =e^{n\left(H\left(Y \mid X_{1}, X_{2}, S_{2}, U, Q\right)+2 \epsilon\right)} e^{-n\left(H\left(Y \mid S_{2}, U, Q, X_{2}\right)-2 \epsilon\right)} \\
& =e^{-n\left(I\left(X_{1} ; Y \mid X_{2}, S_{2}, U, Q\right)-4 \epsilon\right)}
\end{aligned}
$$

where $\widehat{\mathbf{x}}_{1}=\mathbf{x}_{1}\left(\widehat{\mathbf{s}}_{1}, \mathbf{u}, \mathbf{q}\right), \mathbf{x}_{2}=\mathbf{x}_{2}\left(\mathbf{s}_{2}, \mathbf{u}, \mathbf{q}\right)$, and (24) and (25) follow from Lemma 1. It then follows from (23), Lemma 1 and (17) that

$$
\begin{aligned}
& \mathbb{E}_{\mathcal{C}_{1}, \mathcal{C}_{2}}\left[\operatorname{Pr}\left\{E_{1} \mid A_{0}\right\}\right] \\
& \leq\left|T_{\epsilon}^{(n)}\left(S_{1} \mid \mathbf{s}_{2}\right)\right| e^{-n\left(I\left(X_{1} ; Y \mid X_{2}, S_{2}, U, Q\right)-4 \epsilon\right)} \\
& \leq e^{n\left(H\left(S_{1} \mid S_{2}\right)+2 \epsilon\right)} e^{-n\left(I\left(X_{1} ; Y \mid X_{2}, S_{2}, U, Q\right)-4 \epsilon\right)} \\
& \quad=e^{-n\left(I\left(X_{1} ; Y \mid X_{2}, S_{2}, U, Q\right)-H\left(S_{1} \mid S_{2}\right)-6 \epsilon\right)} \\
& \leq e^{-n \epsilon} \leq \epsilon_{0},
\end{aligned}
$$

for $n$ sufficiently large. Similarly, we can bound using (18)

$$
\mathbb{E}_{\mathcal{C}_{1}, \mathcal{C}_{2}}\left[\operatorname{Pr}\left\{E_{2} \mid A_{0}\right\}\right] \leq \epsilon_{0},
$$

for $n$ sufficiently large.

It remains to bound $\mathbb{E}_{\mathcal{C}_{1}, \mathcal{C}_{2}}\left[\operatorname{Pr}\left\{E_{3} \mid A_{0}\right\}\right]$. Write

$$
\begin{aligned}
& \mathbb{E}_{\mathcal{C}_{1}, \mathcal{C}_{2}}\left[\operatorname{Pr}\left\{E_{3} \mid A_{0}\right\}\right] \leq \sum_{\widetilde{\mathbf{s}}_{1} \neq \mathbf{s}_{1}, \widetilde{\mathbf{s}}_{2} \neq \mathbf{s}_{2}:\left(\widetilde{\mathbf{s}}_{1}, \widetilde{\mathbf{s}}_{2}\right) \in T_{\epsilon}^{(n)}\left(S_{1}, S_{2}\right)} \\
& \mathbb{E}_{\mathcal{C}_{1}, \mathcal{C}_{2}}\left[\operatorname{Pr}\left\{\mathbf{v}_{2} \in T_{\epsilon}^{(n)} \mid A_{0}\right\}\right],
\end{aligned}
$$

where $\mathbf{v}_{2}=\left(\widetilde{\mathbf{s}}_{1}, \widetilde{\mathbf{s}}_{2}, \mathbf{u}, \mathbf{q}, X_{1}^{n}\left(\widetilde{\mathbf{s}}_{1}, \mathbf{u}, \mathbf{q}\right), X_{2}^{n}\left(\widetilde{\mathbf{s}}_{2}, \mathbf{u}, \mathbf{q}\right), Y^{n}\right)$ and

$$
\begin{aligned}
& \mathbb{E}_{\mathcal{C}_{1}, \mathcal{C}_{2}}\left[\operatorname{Pr}\left\{\mathbf{v}_{2} \in T_{\epsilon}^{(n)} \mid A_{0}\right\}\right] \\
& \leq \sum_{\mathcal{X}_{1}^{n} \times \mathcal{X}_{2}^{n}} P_{X_{1}^{n} \mid S_{1}^{n} U^{n} Q^{n}}\left(\widetilde{\mathbf{x}}_{1} \mid \widetilde{\mathbf{s}}_{1}, \mathbf{u}, \mathbf{q}\right) P_{X_{2}^{n} \mid S_{2}^{n} U^{n} Q^{n}}\left(\widetilde{\mathbf{x}}_{2} \mid \widetilde{\mathbf{s}}_{2}, \mathbf{u}, \mathbf{q}\right) \\
& \quad \sum_{\mathbf{y} \in T_{\epsilon}^{(n)}\left(Y \widetilde{\mathbf{s}}_{1}, \widetilde{\mathbf{s}}_{2}, \mathbf{u}, \mathbf{q}, \widetilde{\mathbf{x}}_{1}, \widetilde{\mathbf{x}}_{2}\right)} P_{Y^{n} \mid U^{n} Q^{n}(\mathbf{y} \mid \mathbf{u}, \mathbf{q})} \\
& \leq\left|T_{\epsilon}^{(n)}\left(Y \mid \widetilde{\mathbf{s}}_{1}, \widetilde{\mathbf{s}}_{2}, \mathbf{u}, \mathbf{q}, \widetilde{\mathbf{x}}_{1}, \widetilde{\mathbf{x}}_{2}\right)\right| e^{-n(H(Y \mid U, Q)-2 \epsilon)} \\
& \leq e^{n\left(H\left(Y \mid U, Q, X_{1}, X_{2}\right)+2 \epsilon\right)} e^{-n(H(Y \mid U, Q)-2 \epsilon)} \\
& =e^{-n\left(I\left(X_{1}, X_{2} ; Y \mid U, Q\right)-4 \epsilon\right)}
\end{aligned}
$$

where $\widetilde{\mathbf{x}}_{1}=\mathbf{x}_{1}\left(\widetilde{\mathbf{s}}_{1}, \mathbf{u}, \mathbf{q}\right)$ and $\widetilde{\mathbf{x}}_{2}=\mathbf{x}_{2}\left(\widetilde{\mathbf{s}}_{2}, \mathbf{u}, \mathbf{q}\right)$, and (30) and (31) follow Lemma 1. It then follows that,

$$
\begin{aligned}
& \mathbb{E}_{\mathcal{C}_{1}, \mathcal{C}_{2}}\left[\operatorname{Pr}\left\{E_{3} \mid A_{0}\right\}\right] \\
& \quad \leq \sum_{\widetilde{\mathbf{s}}_{1} \neq \mathbf{s}_{1}, \widetilde{\mathbf{s}}_{2} \neq \mathbf{s}_{2}:\left(\widetilde{\mathbf{s}}_{1}, \widetilde{\mathbf{s}}_{2}\right) \in T_{\epsilon}^{(n)}\left(S_{1}, S_{2}\right)} e^{-n\left(I\left(X_{1}, X_{2} ; Y \mid U\right)-4 \epsilon\right)} \\
& \leq\left|T_{\epsilon}^{(n)}\left(S_{1}, S_{2}\right)\right| e^{-n\left(I\left(X_{1}, X_{2} ; Y \mid U\right)-4 \epsilon\right)} \\
& \leq e^{n\left(H\left(S_{1}, S_{2}\right)+2 \epsilon\right)} e^{-n\left(I\left(X_{1} ; Y \mid X_{2}, S_{2}, U\right)-4 \epsilon\right)} \\
& \quad=e^{-n\left(I\left(X_{1} ; Y \mid X_{2}, S_{2}, U\right)-H\left(S_{1}, S_{2}\right)-6 \epsilon\right)} \\
& \leq e^{-n \epsilon} \leq \epsilon_{0}
\end{aligned}
$$

for $n$ sufficiently large. Now plugging (22), (27), (28), and (32) back into (21), and setting $\epsilon_{0}=\frac{\epsilon_{1}}{4}$, we see that $\mathbb{E}_{\mathcal{C}_{1}, \mathcal{C}_{2}}\left[P_{e}^{(n)}\right] \leq$ $\epsilon_{1}$ for $n$ sufficiently large. 


\section{Bounding $\mathbb{E}_{\mathcal{C}_{1}, \mathcal{C}_{2}}\left[P_{i}^{(n)}\right]$}

Since the encoding is separately performed, Encoder 1 is independent of $\mathcal{C}_{2}$. Thus it suffices to show that $\mathbb{E}_{\mathcal{C}_{1}}\left[P_{1}^{(n)}\right] \leq$ $\epsilon_{1}$ for $n$ sufficiently large.

Clearly, if $\left(\mathbf{s}_{1}, \mathbf{u}, \mathbf{x}_{1}\right) \in T_{\epsilon}^{(n)}\left(S_{1}, U, X_{1} \mid \mathbf{q}\right)$, then

$$
\frac{1}{n} d_{1}\left(\mathbf{u}, \mathbf{x}_{1}\left(\mathbf{s}_{1}, \mathbf{u}, \mathbf{q}\right)\right) \leq \mathbb{E}\left[d_{1}\left(U, X_{1}\right)\right]+\epsilon \leq D_{1}+\epsilon
$$

for $n$ sufficiently large, where the first inequality follows from the definition of strong typicality and the second inequality follows from (20). According to Lemma 1,

$$
\begin{aligned}
\mathbb{E}_{\mathcal{C}_{1}}\left[P_{1}^{(n)}\right] \leq & \sum_{\left(T_{\epsilon}^{(n)}\left(S_{1}, U \mid \mathbf{q}\right)\right)^{c}} P_{S_{1}^{n} U^{n}}\left(\mathbf{s}_{1}, \mathbf{u}\right) \\
& +\sum_{T_{\epsilon}^{(n)}\left(S_{1}, U \mid \mathbf{q}\right)} P_{S_{1}^{n} U^{n}}\left(\mathbf{s}_{1}, \mathbf{u}\right) \mathbb{E}_{\mathcal{C}_{1}} \Phi\left\{\mathbf{v}_{3} \notin T_{\epsilon}^{(n)}\right\} \\
\leq & \frac{\epsilon_{1}}{2}+\frac{\epsilon_{1}}{2}=\epsilon_{1}
\end{aligned}
$$

for $n$ sufficiently large, where $\mathbf{v}_{3}=\left(\mathbf{s}_{1}, \mathbf{u}, \mathbf{q}, X_{1}^{n}\left(\mathbf{s}_{1}, \mathbf{u}, \mathbf{q}\right)\right)$ and $\Phi(A)$ is the indicator function of the event $A$.

\section{Completing the Proof}

As we mentioned in the beginning of the section,

$$
\mathbb{E}_{\mathcal{C}_{1}, \mathcal{C}_{2}}\left\{P_{e}^{(n)}+P_{1}^{(n)}+P_{2}^{(n)}\right\} \leq 3 \epsilon_{1},
$$

implies that there exists a pair of codes $\left(\mathcal{C}_{1}, \mathcal{C}_{2}\right)$ such that $P_{e}^{(n)} \leq 3 \epsilon_{1}, P_{1}^{(n)} \leq 3 \epsilon_{1}, P_{2}^{(n)} \leq 3 \epsilon_{1}$ are simultaneously satisfied for $n$ sufficiently large. Furthermore, if $P_{i}^{(n)} \leq 3 \epsilon_{1}$, we have

$$
\frac{1}{n} \mathbb{E}\left[d_{i}\left(U^{n}, X_{i}^{n}\right)\right] \leq\left(D_{i}+\epsilon\right)+d_{i}^{\max } P_{i}^{(n)} \leq D_{i}+\delta_{i},
$$

as $n \rightarrow \infty$, by setting $\delta_{i}=\epsilon+3 \epsilon_{1} d_{i}^{\text {max }}$. Thus the distortion constraint is satisfied. This completes the proof of Theorem 1 .

\section{CONCLUding REMARKS}

In this work we derive a sufficient condition with singleletter characterizations for hiding correlated sources against MAC attacks. An uncomputable (and somewhat trivial) outer bound (converse condition) can be easily formulated by applying Fano's inequality in terms of a sequence of $n$-dimensional joint distributions. We are currently studying the embedding of correlated sources with joint embedding-compression rate constraints. Our next step is to answer the question: when $\left(S_{1}, S_{2}\right)$ are $\left(D_{1}, D_{2}\right)$-admissible with respect to $W_{Y \mid X_{1} X_{2}}$, what is the compression limit for the sources $\left(S_{1}, S_{2}\right)$ and $U$ ?

\section{APPENDIX}

Proof of the Case of Parallel Attack Channels: When $W_{Y \mid X_{1} X_{2}}=W_{Y_{1} \mid X_{1}} \times W_{Y_{2} \mid X_{2}}$, we see that (10)-(12) imply (1)-(4). In fact, if the maximums in (10)-(12) are achieved by $P_{X_{1} \mid U}^{*}\left(x_{1} \mid u\right)$ and $P_{X_{2} \mid U}^{*}\left(x_{2} \mid u\right)$, then simply letting $|\mathcal{Q}|=1$, $P_{X_{1} \mid S_{1} U}\left(x_{1} \mid s_{1}, u\right)=P_{X_{1} \mid U}^{*}\left(x_{1} \mid u\right)$ and $P_{X_{2} \mid S_{2} U}\left(x_{2} \mid s_{2}, u\right)=$ $P_{X_{2} \mid U}^{*}\left(x_{2} \mid u\right)$, we see that with this choice,

$I\left(X_{1} ; Y \mid X_{2}, S_{2}, U, Q\right)=I\left(X_{1} ; Y_{1} \mid S_{2}, U, Q\right)=I\left(X_{1} ; Y_{1} \mid U\right)$
Similarly,

$$
=\max _{\mathbb{E}\left[d_{1}\left(U, X_{1}\right)\right] \leq D_{1}} I\left(X_{1} ; Y_{1} \mid U\right) .
$$

$$
I\left(X_{2} ; Y \mid X_{1}, S_{1}, U, Q\right)=\max _{\mathbb{E}\left[d_{2}\left(U, X_{2}\right)\right] \leq D_{2}} I\left(X_{2} ; Y_{2} \mid U\right),
$$

and

$$
\begin{aligned}
& I\left(X_{1}, X_{2} ; Y_{1}, Y_{2} \mid U, Q\right) \\
& \quad=\max _{\mathbb{E}\left[d_{1}\left(U, X_{1}\right)\right] \leq D_{1}} I\left(X_{1} ; Y_{1} \mid U\right)+\max _{\mathbb{E}\left[d_{2}\left(U, X_{2}\right)\right] \leq D_{2}} I\left(X_{2} ; Y_{2} \mid U\right) .
\end{aligned}
$$

We next show that (1)-(4) imply (10)-(12). We only need to show that for any $P_{X_{1} \mid S_{1} U Q}$ satisfying $\mathbb{E}\left[d_{1}\left(U, X_{1}\right)\right]<D_{1}$, the right hand side of (1) is upper bounded by (10). Since $\left(Q, S_{1}, U\right) \rightarrow X_{1} \rightarrow Y_{1}$ form a Markov chain in this order,

$$
\begin{aligned}
I\left(X_{1} ; Y_{1} \mid U\right) & =H\left(Y_{1} \mid U\right)-H\left(Y_{1} \mid X_{1}, U\right) \\
& \geq H\left(Y_{1} \mid S_{2}, U, Q\right)-H\left(Y_{1} \mid X_{1}, S_{2}, U, Q\right) \\
& =I\left(X_{1} ; Y_{1} \mid S_{2}, U, Q\right) .
\end{aligned}
$$

For any $P_{X_{1} \mid S_{1} U Q}$ satisfying $\mathbb{E}\left[d_{1}\left(U, X_{1}\right)\right]<D_{1}$, set

$$
\widehat{P}_{X_{1} \mid U}\left(x_{1} \mid u\right)=\sum_{\mathcal{S}_{1} \times \mathcal{Q}} P_{S_{1}}\left(s_{1}\right) P_{Q}(q) P_{X_{1} \mid S_{1} U Q}\left(x_{1} \mid s_{1}, u, q\right) .
$$

Under the corresponding $\widehat{P}_{X_{1} \mid U}\left(x_{1} \mid u\right)$, we have

$$
\begin{aligned}
I\left(X_{1} ; Y_{1} \mid U, S_{2}, Q\right) & \leq I\left(X_{1} ; Y_{1} \mid U\right) \\
& \leq \max _{\mathbb{E}\left[d_{1}\left(U, X_{1}\right)\right] \leq D_{1}} I\left(X_{1} ; Y_{1} \mid U\right) .
\end{aligned}
$$

We can similarly show that (2)-(3) imply (11)-(12).

\section{REFERENCES}

[1] A. S. Cohen and A. Lapidoth, "The Gaussian watermarking game," IEEE Trans. Inform. Theory, vol. 48, no. 6, pp. 1639-1667, Jun. 2002.

[2] D. Karakos and A. Papamarcou, "A relationship between quantization and watermarking rates in the presence of additive Gaussian attacks," IEEE Trans. Inform. Theory, vol. 49, pp. 1970-1982, August 2003.

[3] A. Maor and N. Merhav, "On joint information embedding and lossy compression in the presence of a memoryless attack," IEEE Trans. Inform. Theory, vol. 51, pp. 3166-3175, Sep. 2005.

[4] P. Moulin and J. O'Sullivan, "Information-theoretic analysis of information hiding," IEEE Trans. Inform. Theory, vol. 49, no. 3, pp. 563-593, Mar. 2003.

[5] F. A. P. Petitcolas, R. J. Anderson and M. G. Kuhn, "Information hiding - a survey," Proc. IEEE, vol. 87, no. 7, pp. 1062-1078, Jul. 1999.

[6] A. Somekh-Baruch and N. Merhav, "On the capacity game of public watermarking systems," IEEE Trans. Inform. Theory, vol. 50, no. 3, pp. 511-524, Mar. 2004.

[7] E.-H. Yang and W. Sun, "On watermarking and compression rates of joint compression and private watermarking systems with abstract alphabets," Proc. of the 2005 Canadian Workshop on Information Theory, Montreal, Quebec, Canada, June 5-8, pp. 296-299, 2005.

[8] E.-H. Yang and W. Sun, "On information embedding when watermarks and covertexts are correlated"Proc. of IEEE IEEE Int'l. Symp. Inform. Theory, pp. 346-350, Seattle, USA, 2006.

[9] T. M. Cover, A. El Gamal and M. Salehi, "Multiple access channels with arbitrarily correlated sources," IEEE Trans. Inform. Theory, vol. 26, no. 6, Nov. 1980.

[10] R. Ahlswede and T. S. Han, "On source coding with side information via a multiple-access channel and related problems in multi-user information theory," IEEE Trans. Inform. Theory, vol. 29, May 1983.

[11] D. Slepian and J. K. Wolf, "Noiseless coding of correlated information sources," IEEE Trans. Inform. Theory, vol. 19, pp. 471-480, July 1973.

[12] D. Slepian and J. K. Wolf, "A coding theorem for multiple access channels with correlated sources," Bell Syst. Tech. Journal, vol. 52, pp. 1037-1076, 1973.

[13] T. M. Cover and J. A. Thomas, Elements of Information Theory, 2nd Ed., Wiley, 2006. 